\title{
3D Recovery with Simultaneous Accuracy Estimation and the Following Mesh Refinement
}

\author{
Natalya V. Sveshnikova, Dmitry V. Yurin
}

\begin{abstract}
This paper presents a feature-based 3D recovery system. First the scene model is recovered by the factorization algorithms from the digital images sequence. This approach also provides the result error estimation. Next the preliminary mesh is refined as follows. We choose an image from the sequence and search for the additional features using the Harris corner detector. Then we choose the second image from the sequence and estimate the correspondences positions for the additional features using the small set of reliable features. Finally estimations are defined more accurately by the Kanade-Lucas tracker.
\end{abstract}

Index Terms-3D Recovery by Factorization Algorithms, epipole geometry, Harris corner detector, Kanade-Lucas Tracker.

\section{INTRODUCTION}

$\mathrm{T}$ HE 3D recovery problem of the real scenes recovery has different application domains and is intensively investigated [1]-[8]. Though the high-end toolkit for the manual 3D model development is available nowadays, it is a laborious method if we want to get a model of the real object even if the shape is rather simple. That is why the automated methods of 3D recovery have a good future prospect.

Two general sorts can be separated out among the great amount of different 3D recovery methods. The algorithms of the first type recover the dense 3D model as for example the standard stereo [1], [2] does. In contrast the algorithms of the second type recover the 3D model of the scene as the set of feature points [3]-[7]. The feature-based recovery is typical for the group of shape from motion algorithms and the widebase stereo. The standard stereo supposes that the input images are rectified and the relative shift is small. It also requires the disparities search range to be defined. It is a crucial parameter as the understated range lead to the wrong result and the overstated range appreciably slows down the

Manuscript received May 25, 2007. This work was supported in part by the Russian Foundation for Basic Research under Grant 06-01-00789, 05-07-90345, 05-07-90390.

N. V. Sveshnikova is a Ph.D. student at Moscow Institute of Physics and Technology, Department of Physical and Quantum Electronics, sub-faculty of SIM. Her contact email is sveshnikova_n@list.ru.

Dmitry V. Yurin, PhD, is a chief specialist in FGUP RPE OPTECS (The Federal State Unitary Enterprise Research and Production Enterprise "Optoelectronic Complexes and Systems") and a senior researcher at Moscow State University, Department of Computational Mathematics and Cybernetics. His contact email is yurin_d@inbox.ru. computation. In contrast the wide-based stereo such as [7] and the shape from motion approaches such as factorization algorithms [3]-[6] require the set of corresponding feature points detected and tracked in the input images. Practically these approaches are more perspective than the standard stereo since the wide base and/or the considerable amount of input images increase the recovery accuracy. Moreover one can use the typical photos and videos as the input images. The preliminary recovery can be obtained even if the number of feature points is rather small $(\sim 10)$, though in this case the triangulation mesh is insufficient to model the real scene surface.

Nevertheless the preliminary recovery gives a coarse approximation of the real scene form even if the number of feature points is small. This information can be used for the further refinement. Using the available reliable correspondences we can estimate the position and constrain the search range of the new correspondences.

The incremental algorithm of the constrained search of correspondences was introduced in [8]. The main idea is to constrain at most the search range of the corresponding point. If the set of reliable correspondences is given, one can use Delaunay triangulation to part the first image into the set of triangles which is extends to the second image. Thus a set of corresponding triangles on two images is created. The same set of reliable correspondences can be used to compute the epipole geometry. So for every feature point they search for the corresponding one in the piece of epipolar line constrained by the corners of the corresponding triangles/

In this paper we introduce the approach that also use the triangulation of reliable correspondences to constrain the search range of new correspondences, however it proposes the different method of position and search range estimation. In contrast to [8] we suppose the shape from motion problem is solved already. We use the mesh built on the small set of reliable features and the recovered positions and orientations of the cameras to form hypotheses of the corresponding features on the second image. This is more severe constraint then a search range given by the intersection of the mesh triangle and the epipolar line.

In the next section we describe the structure of our approach. Several steps of the system are specified in the following sections. Finally we present the results of the 3D recovery of the real scene. 


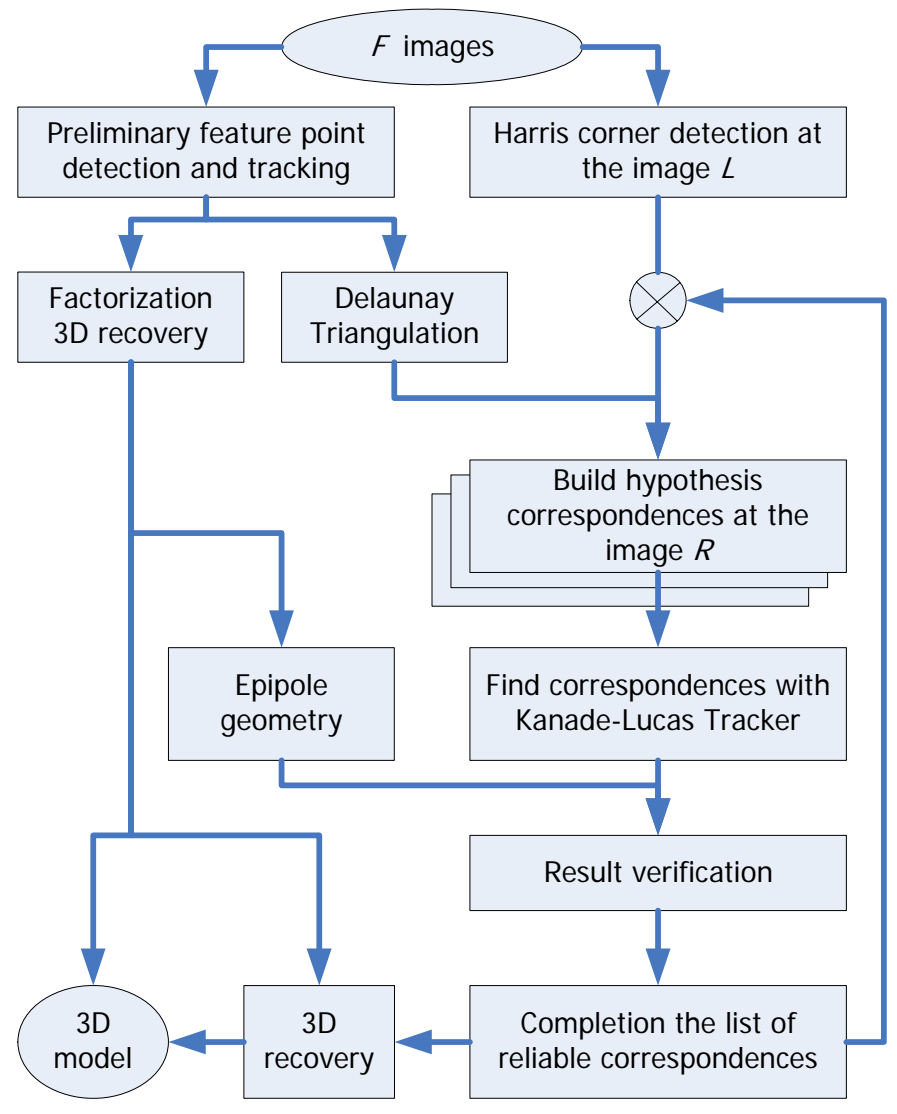

Fig. 1. The structure of the 3D recovery system.

\section{THE APPROACH REVIEW}

Let the $F$ images $(F>5)$ of the static scene are given. There are no specific requirements to the calibration or the quality of the camera. Also let the small number $(P \sim 10 \div 20)$ of feature points are detected and tracked through the whole set of digital images. The simplest way is to set the feature points manually. These source data are enough to recover the 3D positions of the feature points $\mathbf{S}=\left\{\vec{s}_{p}\right\}$ by the factorization algorithms [3]-[6], as well as to compute all cameras positions $\mathbf{t}=\left\{\vec{t}_{f}\right\}$, orientations $\mathbf{R}=\left\{\vec{i}_{f}, \vec{j}_{f}, \vec{k}_{f}\right\}$, the focal length $g$ and to give the estimation of the result accuracy [5], [6]. For more details about the factorization algorithms and their accuracy, see the next section.

We perform the Delaunay triangulation to build the preliminary mesh that models the scene surface by the set of large plane pieces according to the result of triangulation. To refine the model we have to detect more features and to track their correspondence.

Suppose the scene is a piecewise smooth surface. Though the preliminary mesh may not match up the real shape of the scene we consider that the mesh gives a reliable approximation of the scene shape in the neighborhood of the triangle vertices.

We choose the image $L$ from the initial set of digital images and use it to detect features by the Harris corner detector [9]. Then for each feature we form one or more hypothesizes of the corresponding point position at the second chosen image $R$. The final search of the correspondence is performed by the Kanade-Lucas tracker [10].

The tracked correspondences must satisfy to the epipole constraints. The epipole geometry is computed from the cameras positions and orientations recovered by the factorization algorithms. We define the constraint for the new correspondence as follows. The distance between the point $\vec{X}_{R}$ at the second image $R$ and the epipolar line

$$
\vec{X}_{L}^{T} \mathbf{F} \vec{x}=0
$$

must be smaller then the error of the epipolar line, which estimation is specified in the section IV. Here we denoted fundamental matrix as $\mathbf{F}$ and the corresponding point at the first image as $\vec{x}_{L}$.

At last we add new reliable correspondences to the initial set of features and build a new mesh. Using the cameras positions and orientations recovered by the factorization algorithms we can compute the 3D positions of new features and supplement the 3D model of the scene. Or again we can try to add new correspondences using the refined mesh. The structure of the described approach is sketched out at the Fig. 1

\section{SHAPE FROM MOTION BY THE FACTORIZATION ALGORITHMS}

Here we consider that $3 \mathrm{D}$ scene is not deformed during the shooting. In other words the scene is a solid object or several relatively motionless solid objects.

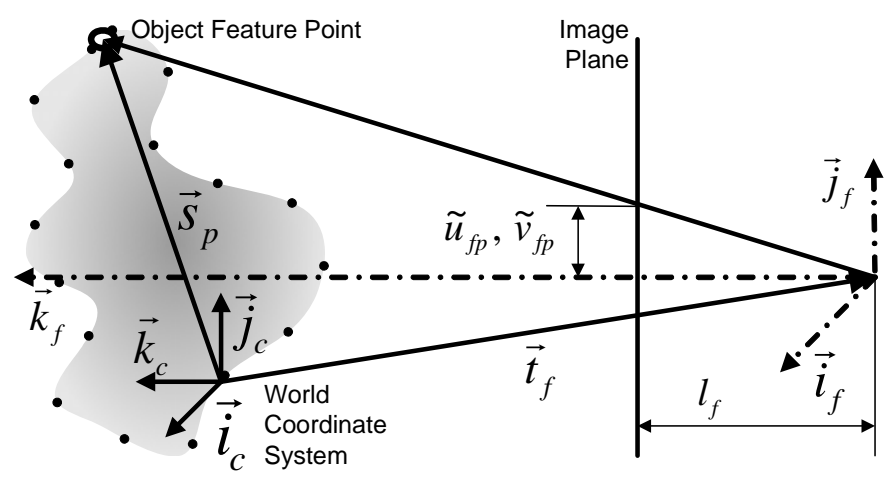

Fig. 2. Statement of the 3D scene recovery problem.

The 3D coordinates of the $p$ th point are related to their projections to the image plane (Fig. 2) by the well-known equations of the perspective projection [2]-[5]:

$$
u_{f p}=g_{f} \frac{\vec{i}_{f}\left(\vec{s}_{p}-\vec{t}_{f}\right)}{\vec{k}_{f}\left(\vec{s}_{p}-\vec{t}_{f}\right)} ; \quad v_{f p}=g_{f} \frac{\vec{j}_{f}\left(\vec{s}_{p}-\vec{t}_{f}\right)}{\vec{k}_{f}\left(\vec{s}_{p}-\vec{t}_{f}\right)}
$$


where $g_{f}=\frac{l_{f}}{\tilde{u}_{f}^{e} N}, \quad u_{f p}=\frac{\tilde{u}_{f p}}{\tilde{u}_{f}^{e} N}, \quad v_{f p}=\frac{\tilde{v}_{f p}}{\tilde{u}_{f}^{e} N}$

Here we denoted the orthonormal basis vectors specifying the right coordinate system associated with the $f$ th camera as $\vec{i}_{f}, \vec{j}_{f}, \vec{k}_{f} ; l_{f}$ is the focal length, $\tilde{u}_{f}^{e}$ is the size of the pixel on the optical sensor, $N$ is the image width in pixels. Introducing the notation $Z_{f}^{\prime}=-\vec{k}_{f} \vec{t}_{f} / g_{f}$ and moving the denominator to the left part of (2) we get the following expression.

$$
\begin{gathered}
\left\{\begin{array}{l}
\left(1+\frac{1}{g} \frac{\vec{k}_{f} \vec{s}_{p}}{Z_{f}^{\prime}}\right) u_{f p}=\vec{m}_{f} \vec{s}_{p}+t_{X f} \\
\left(1+\frac{1}{g} \frac{\vec{k}_{f} \vec{s}_{p}}{z_{f}^{\prime}}\right) v_{f p}=\vec{n}_{f} \vec{s}_{p}+t_{Y f}
\end{array}\right. \\
\text { where }\left\{\begin{array}{l}
\vec{m}_{f}=\vec{i}_{f} / z_{f}^{\prime} \\
\vec{n}_{f}=\vec{j}_{f} / z_{f}^{\prime}
\end{array},\left\{\begin{array}{l}
t_{X f}=-\vec{i}_{f} \vec{t}_{f} / z_{f}^{\prime} \\
t_{Y f}=-\vec{j}_{f} \vec{t}_{f} / z_{f}^{\prime}
\end{array}\right.\right.
\end{gathered}
$$

Assuming that $\vec{k}_{f} \vec{s}_{p} / z_{f}<<1$ we obtain the following formulas for the scaled orthographic projection (SOP) approximation.

$$
u_{f p}=\vec{m}_{f} \vec{s}_{p}+t_{X f} \quad v_{f p}=\vec{n}_{f} \vec{s}_{p}+t_{Y f}
$$

The collection of equations (4) for $P$ points and $F$ images can be represented as a matrix equation.

$$
\mathbf{W}=\mathbf{M S}+\mathbf{T} ; \quad \mathbf{W}^{\prime}=\mathbf{W}-\mathbf{T}
$$

Where $\mathbf{W}$ is a $2 F \times P$ matrix formed by the feature points coordinates on the images planes, matrix $\mathbf{M}$ contains the information about cameras positions and orientations and the matrix $\mathbf{S}$ consist of the 3D features coordinates. Hence $\mathbf{W}^{\prime}$ can be represented as the product of matrices the rank of which does not exceed $\zeta$ which is equal to 3 , or 2 , or 1 for a 3D, plane, and one-dimensional objects, respectively [3]. Therefore, the rank of $\mathrm{W}^{\prime}$ also does not exceed this value.

The factorization problem is solved by the singular-value decomposition (SVD) of the matrix $\mathrm{W}^{\prime}$. The idea is to choose three largest singular values $\sigma_{i}, i=\{x, y, z\}$ and the corresponding singular vectors [5].

To solve the problem in the perspective projection we have to work with (3). Considering the focal length is constant for the whole set of images, i.e. $g_{f}=g$, we can represent (3) as a matrix equation. This equation is similar to (5), but its left part contains the adding, that is the correction associated with the perspective distortions.

$$
\begin{gathered}
\tilde{\mathbf{W}}=\mathbf{M S}+\mathbf{T} \text {, where } \tilde{\mathbf{W}}=\mathbf{W}+\xi \mathbf{W}_{2}, \\
\xi=\frac{1}{g}, \mathbf{W}_{2}=\left\{\begin{array}{l}
\vec{k}_{f} \vec{s}_{p} u_{f p} / z_{f}^{\prime} \\
\vec{k}_{f} \vec{s}_{p} v_{f p} / z_{f}^{\prime}
\end{array}\right\}
\end{gathered}
$$

We propose to solve (6) iteratively by successively refining the focal length and using the results of previous step of the iterations to compute the adding $\mathbf{W}_{2}$. The detailed description of the iterative algorithm in perspective projection (IPP) is given in [5].

The 3D recovery algorithms SOP and IPP work under the conditions of the excessive information. In the presence of noise, the constraints (2)-(4) may be violated; however, these violations are not arbitrary and obey certain statistical laws. This provides the algorithms belonging to the considered group with the unique feature: simultaneously with the shape and motion recovery, the errors of the results being obtained can be estimated. Moreover, it turns out that the error estimates can be obtained in the framework of the same technique (singular value decomposition).

$$
\begin{gathered}
\varepsilon_{\text {shape }}=\sigma_{n} \sqrt{\sum_{i=\{x, y, z\}} \frac{1}{\left\|\vec{M}_{i}\right\|^{2}\left\|\vec{S}_{i}\right\|^{2}}} \\
\varepsilon_{\text {rotation }}=\frac{\sqrt{2} \sigma_{n}}{\|\mathbf{M}\|} \sqrt{\sum_{i=\{x, y, z\}} \frac{1}{\left\|\vec{S}_{i}\right\|^{2}}} \\
\varepsilon_{\text {camera } z}=\frac{\sigma_{n}}{\sqrt{\sum_{i=\{x, y, z\}} \sigma_{i}^{2}}}
\end{gathered}
$$

Here $\|\cdot\|$ is a Frobenius norm for matrices [11], $\sigma_{n}$ is a noise-induced singular value, vectors $\vec{M}_{i}$ are the columns of matrix $\mathbf{M}$ and the vectors $\vec{S}_{i}$ are the rows of matrix $\mathbf{S}$. The detailed derivation of (7) is given in [6]. The derivation does not use any additional constraints on the shape of the scene or the way of shooting. Therefore the result in [6] is more general than the simple estimates in [5] where the scene considered to be of a small depth.

\section{EPIPOLAR GEOMETRY}

The basic result of the epipolar geometry is the linear relation between the homogeneous coordinates $\vec{x}_{L}$ and $\vec{x}_{R}$ of 3D scene point projection on the planes of the two images $L$ and $R$ respectively. 


$$
\vec{x}_{L}^{T} \mathbf{F} \vec{x}_{R}=0 \quad, \quad \operatorname{rank} \mathbf{F}=2
$$

Components of the fundamental matrix $\mathbf{F}$ (FM) can be represented as follows [12].

$$
F_{m n}=e_{c d a}\left(\vec{t}_{R}-\vec{t}_{L}\right)_{d} r_{m a} r_{n c}^{\prime}, m, n=1 . .3
$$

Where $e_{c d a}$ is a unit antisymmetric Levi-Civita tensor of rank $3, \vec{t}_{L}, \vec{t}_{R}$ are the positions of cameras, $r_{m a}, r_{n c}^{\prime}$ are the components of matrices $\left\{\vec{i}_{L}, \vec{j}_{L}, \vec{k}_{L}\right\},\left\{\vec{i}_{R}, \vec{j}_{R}, \vec{k}_{R}\right\}$, which are the matrices of camera orientations. The expression (9) is a mixed product of the translation vector $\left(\vec{t}_{R}-\vec{t}_{L}\right)$ and the rows of the respective orientation matrices [6].

In the context of the considered problem fundamental matrix is computed by the results of factorization algorithms. Generally the state of the problem solved by the factorization algorithms consider the vectors $\vec{k}_{L}$ and $\vec{k}_{R}$ are pointed to the object centre of mass, since the factorization algorithms require the visibility of all features on the whole set of images. The point of origin is also placed to the object centre of mass. In that case the vectors $\vec{t}_{L}, \vec{t}_{R}$ can be represented as follows.

$$
\vec{t}_{L}=-z_{L} \vec{k}_{L}, \quad \vec{t}_{R}=-Z_{R} \vec{k}_{R}
$$

Where $Z_{L}$ and $Z_{R}$ are the distances between the cameras and the object centre of mass.

Using (10) we can expand the expression for the each component of fundamental matrix.

$\mathbf{F}=\left(\begin{array}{ccc}z_{R}\left(\vec{j}_{R}, \vec{i}_{L}\right)+z\left(\vec{j}_{L}, \vec{i}_{R}\right) & -z_{R}\left(\vec{i}_{R}, \vec{i}_{L}\right)+z\left(\vec{j}_{L}, \vec{j}_{R}\right) & z_{L}\left(\vec{j}_{L}, \vec{k}_{R}\right) \\ z_{R}\left(\vec{j}_{R}, \vec{j}_{L}\right)-z\left(\vec{i}_{L}, \vec{i}_{R}\right) & -z_{R}\left(\vec{i}_{R}, \vec{j}_{L}\right)-z\left(\vec{i}_{L}, \vec{j}_{R}\right) & -z_{L}\left(\vec{i}_{L}, \vec{k}_{R}\right) \\ -z_{R}\left(\vec{j}_{R}, \vec{k}_{L}\right) & -z_{R}\left(\vec{i}_{R}, \vec{k}_{L}\right) & 0\end{array}\right)$

Deriving the differential of the fundamental matrix components (11) and approximating them by the increments [13] we get the estimation for the fundamental matrix error.

$$
\Delta \mathbf{F}=\varepsilon_{\text {camera }_{-} z} \mathbf{F}+\varepsilon_{\text {rotation }} \boldsymbol{\Phi}
$$

Where

$$
\boldsymbol{\Phi}=\left(\begin{array}{ccc}
z_{R}+z_{L} & -z_{R}+z_{L} & g_{R} z_{R} \\
z_{R}-z_{L} & -z_{R}-z_{L} & g_{R} z_{R} \\
g_{L} z_{L} & g_{L} z_{L} & 0
\end{array}\right)
$$

Using (12) we can easily estimate the error of the epipolar lines. Let the equation of the epipolar line is defined in general form and denoted as follows.

$$
\vec{A} \vec{x}_{R}=0 \text { or } A_{1} u_{R}+A_{2} v_{R}+A_{3}=0
$$

The coefficient in (14) can be evaluated from (8).

$$
\vec{A}=\vec{x}_{L} \mathbf{F} \text { or } A_{i}=\left(\vec{F}_{i}, \vec{x}_{L}\right)
$$

We denoted the columns of fundamental matrix as vectors $\vec{F}_{i}, i=1 . .3$. Next we use (15) to evaluate the increments of the coefficients of the epipolar line equation considering only matrix $\mathbf{F}$ varies.

$$
\Delta \vec{A}=\vec{x} \Delta \mathbf{F} \text { or } \Delta A_{i}=\left(\Delta \vec{F}_{i}, \vec{x}\right)
$$

As appears from the (16) the epipolar line error depends on the feature point position on the image. To eliminate this we derive the root-mean-square error for the whole image under the assumption that the distribution of features is uniform [13].

$$
\Delta \tilde{A}_{i}=\frac{1}{2 \sqrt{3}}\left|\Delta \vec{F}_{i}\right| \sqrt{\left(1+11 \frac{\Delta F_{3 i}^{2}}{\Delta \vec{F}_{i}^{2}}\right)}
$$

\section{THE HYPOTHESES OF THE CORRESPONDING POINT POSITION}

After the Delaunay triangulation is applied we can part each image into the set of triangle pieces. Consider we have a piece with vertices $\left\{\vec{s}_{1}, \vec{s}_{2}, \vec{s}_{3}\right\}$. Now assume that the corresponding piece of the real 3D scene is a plane. Than means the projections $\left\{\vec{u}_{L 1}, \vec{u}_{L 2}, \vec{u}_{L 3}\right\}$ and $\left\{\vec{u}_{R 1}, \vec{u}_{R 2}, \vec{u}_{R 3}\right\}$ of the considered points on the image planes are related by the transformation $\mathbf{A}$ that can be approximated as affine. The transformation A convert each point in the triangle $\left\{\vec{u}_{L 1}, \vec{u}_{L 2}, \vec{u}_{L 3}\right\}$ on the image $L$ to its corresponding point $\mathbf{A} \vec{x}_{L}$ on the image $R$.

If our assumption is wrong and the chosen piece of the real scene is not plane, then the projections are not related with the affine transformation. But still we can use it in the neighborhood of the triangle vertices.

Each feature point can be a vertex for several triangles of the mesh. Therefore we can form several hypothesizes in the neighborhood of the feature. The better hypothesis of the point position matches reality the less expectancy of tracking the false correspondence. Therefore we track by KanadeLucas only features which hypothesizes differ less then the $1 / 3$ of the tracker window size. 


\section{RESUlTS}

The proposed approach was tested on the real scenes. The results are illustrated by the scenes "Cabin" and "Boxes". For the "Cabin" we manually detected 14 featured points and tracked them through the 9 images of the sequence. For the "Boxes" we manually detected 21 featured points and tracked them through the 21 images of the sequence. At the left part of Fig. 3 the initial sets of the feature points are shown. First scenes were recovered by the iterative algorithm in perspective projection and the linear algorithm in scaled orthographic projection [5], [6]. The error estimates of the results are calculated according to (7) and are given in the Table 1. Since the perspective distortion is significant for the both scenes and the accuracy of IPP is better, for the following operations we use the results of iterative algorithm.

TABLE I

ERRORS OF THE 3D RECOVERY BY THE FACTORIZATION ALGORITHMS

\begin{tabular}{lcccc}
\hline \hline \multirow{2}{*}{ Symbol } & \multicolumn{2}{c}{ Scene "Cabin" } & \multicolumn{2}{c}{ Scene "Boxes" } \\
& SOP & IPP & SOP & IPP \\
\hline$\varepsilon_{\text {shape }}$ & 0,146 & 0,070 & 0,191 & 0,083 \\
$\varepsilon_{\text {rotation }}$ & 0,094 & 0,050 & 0,052 & 0,020 \\
$\varepsilon_{\text {camera } Z}$ & 0,035 & 0,018 & 0,014 & 0,005
\end{tabular}

To build a mesh we apply the Delaunay triangulation. We choose an image from the sequence and triangulate the set of its feature points. The results are shown at the left part of Fig. 3. In spite of the shape simplicity coarse mesh distort the shape of recovered model (right part of the Fig. 3).
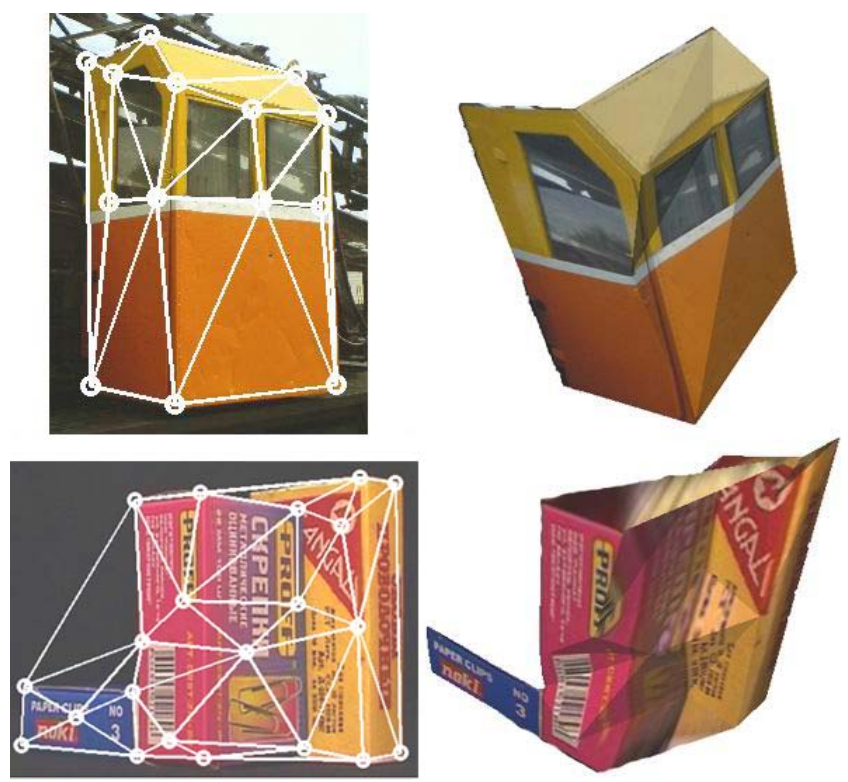

Fig. 3. The result of preliminary recovery of the scenes "Cabin" and "Boxes" by factorization algorithm IPP. Left: the mesh built on the small initial set of feature points. Right: the 3D model of the scene in VRML. Obviously these models poorly matches the real scene shape though it is rather simple.
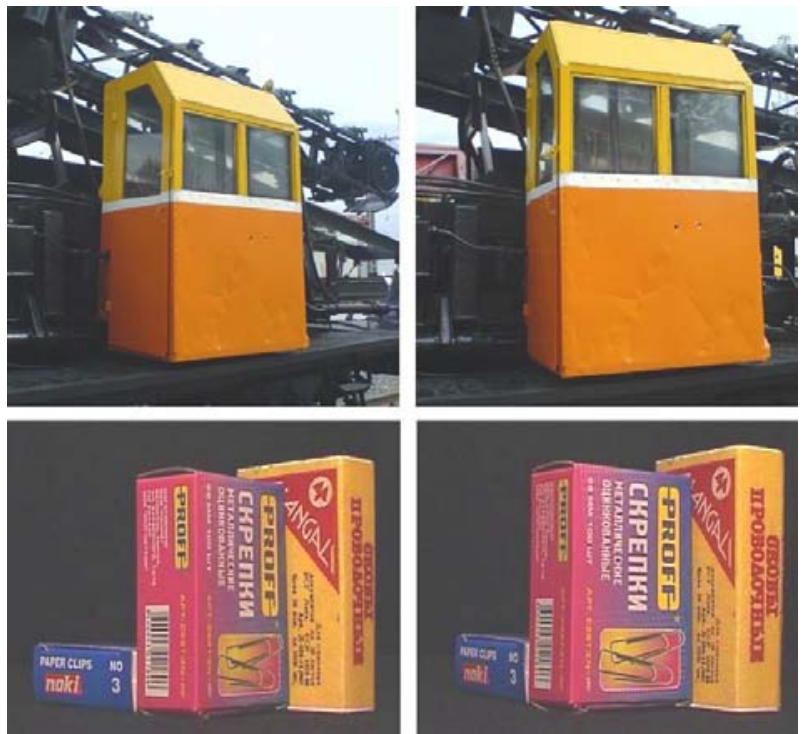

Fig. 4. The pairs of images chosen from the initial sequences for the following mesh refinement.

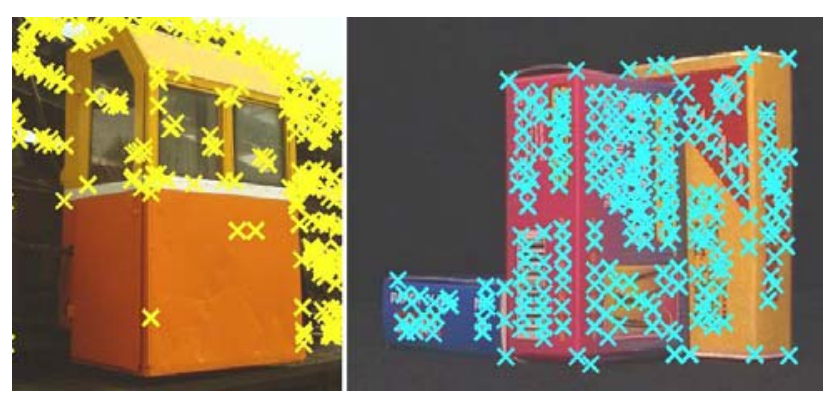

Fig. 5. The Harris corner detector result for the scenes “Cabin” and "Boxes".

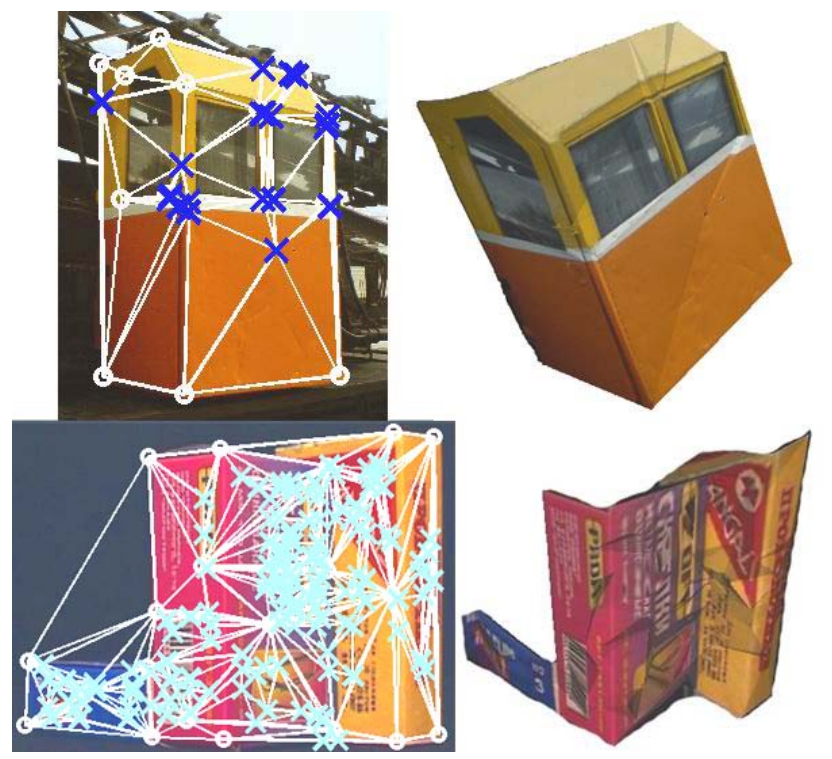

Fig. 6. Final recovery results for the scenes "Cabin" and "Boxes". Left: the mesh built on the sets of feature points. The features of the initial set are marked as $\mathrm{O}$ and the new features tracked by KanadeLucas are marked as X. Right: the 3D models of the scenes "Cabin" and "Boxes" in VRML. One can see that these models match the real scene shapes better than the previous ones. However the accuracy of some correspondences tracked by Kanade-Lucas is not good and it disturbs the surfaces of the 3D models. 
To refine the mesh we manually choose the pair of images from the initial sequence (Fig. 4). We use the left image to detect new features by Harris corner detector [9] which results are shown on Fig. 5.

Then we choose all new feature points, those are placed inside one of the mesh triangles. For each of them we determine the closest reliable correspondence $\vec{u}_{L}$. All the triangles those have the vertex $\vec{u}_{L}$ are used to form hypothesizes. Then we compare different hypothesizes for the same feature. If they differ more then $1 / 3$ of the Kanade-Lucas window size, the feature is not tracked. In this implementation the size of the Kanade-Lucas window is 25 pixels.

Next we track the correspondences by Kanade-Lucas tracker [10].

The tracked correspondences are verified by the epipolar constraints. The fundamental matrix error was estimated according to (12)-(13). The average relative error for the coefficients of fundamental matrix is 0,127 for the scene "Boxes" and 0,164 for the scene "Cabin".

The error of the epipolar line coefficients (14) were estimated according to (17). For the scene "Cabin" it comes to $(0,010 \quad 0,0270,091)$. And for the scene "Boxes" it amounts (0,053 $0,107 \quad 0,040)$.

Finally we get new good correspondences and add them to the set of reliable features. The results are shown at Fig. 6 . The initial set of features marked by "O" and the set of new features marked by " $X$ " are represented at the left side of the Fig. 6 as well as the result of Delaunay triangulation. Totally we add 22 points for the scene "Cabin" and 134 points for the scene "Boxes". The number of new features for the scene "Boxes" is appreciably higher because this scene is more textured than the "Cabin".

The refined 3D models are shown at right side of the Fig. 6 . One can see that the models match the real scene shape better than the previous ones. However the surfaces of the models are distorted. We can see it well at the 3D model of the "Boxes". This occurs because of the false correspondences tracked by Kanade-Lucas tracker. Though we verify the correspondences by epipolar constraints, it does not guarantee that all the false correspondences would be excluded from the final feature set.

Summing up the results of tests one can say that the introduced system demonstrated its efficiency. The results of the iterative factorization algorithm in perspective projection provide a reliable basis for the further result improvement due to the errors estimation. The following mesh refinement enlarges the set of feature points and makes the 3D model more similar to the real scene surface. Nevertheless the problem of false correspondence still needs to be solved.

\section{CONCLUSION}

This paper is devoted to the problem of automated 3D recovery of the real scenes by the set of digital images. We introduced the approach which let to recover the preliminary coarse result by the small set of feature points and to refine it by increasing the number of features. But for the constraint the recovered object is solid the approach does not require any specific condition for the way of shooting as well as it does not use any a priori information about the shape of the scene.

For the preliminary recovery we use the factorization algorithms, those allow to recover the 3D feature coordinates, cameras positions and orientations. Moreover using the excessiveness of information (the number of images $F>5$ ) we succeed in getting the errors estimation (7). The expressions (7) are more general than the similar results in [5]. Estimations (7) do not require any specific way of shooting and use no scene shape restrictions in contrast with [5] where the small depth of scene was considered.

We use the factorization results to compute the epipole geometry and to estimate its errors (13), (17).

To refine the preliminary result we detect the additional features by Harris corner detector and track them by KanadeLucas tracker. The essential peculiarity of the approach is that there no requirement of the small shift between the images that increases the accuracy of the final result. The requirement was obviated due to the small preliminary set of reliable correspondences.

Our system was tested by the real scene image sequences and demonstrated its efficiency. However the system needs some future analysis to solve the problems of false correspondences removal.

\section{ACKNOWLEDGMENT}

We thank professor S. V. Klimenko for his attention and support.

\section{REFERENCES}

[1] V. Kolmogorov and R. Zabih. Computing visual correspondence with occlusions using graph cuts. In International Conference on Computer Vision, Vancouver, Canada, July 2001.

http://www.cs.cornell.edu/rdz/Papers/KZ-ICCV01-tr.pdf.

[2] Michael H. Lin, Carlo Tomasi. Surfaces with Occlusions from Layered Stereo. //IEEE Computer Society Conference on Computer Vision and Pattern Recognition, 2003.

[3] C.J. Poelman, T. Kanade. A Paraperspective Factorization Method for Shape and Motion Recovery. // IEEE Transactions on Pattern Analysis and Machine Intelligence, 1997. -V. 19. -No. 3. -P. 206-218.

[4] Peter Sturm, Bill Triggs. A Factorization Based Algorithm for MultiImage Projective Structure and Motion: //4th European Conference on Computer Vision, Cambridge, England, April 1996, pp 709-720.

[5] N. V. Sveshnikova, D. V. Yurin. Priori and A Posteriori Error Estimates in Recovery of 3D Scenes by Factorization Algorithms. //Programming and Computer Software, Vol. 30, No. 5, 2004, pp. 278-294.

[6] N.V. Sveshnikova, D.V. Yurin The Factorization Algorithms: Results Reliability and Application for the Epipolar Geometry Recovery. // In Conference Proceedings. 16-th International Conference on Computer Graphics and Application GraphiCon'2006 July 1-5, 2006 Novosibirsk Akademgorodok, Russia.

[7] P. Pritchett, A. Zisserman. Wide Baseline Stereo Matching. //Proceedings of the 6th International Conference on Computer Vision, Bombay, India, Jan. 1998. -P. 754-760.

http://www.robots.ox.ac.uk/\%7evgg/publications/html/index.html 
[8] V. Gouet, P. Montesinos and D. Pele. A fast matching method for color uncalibrated images using differential invariants. //In Proceedings of the British Machine Vision Conference, Southampton, 1998.

[9] C. G. Harris and M. Stephens, "A combined corner and edge detector," In Proc. 4th Alvey Vision Conf., Manchester, pages 147-151, 1988.

[10] Carlo Tomasi, Takeo Kanade. Shape and Motion from Image Streams: a Factorization Method, Part 3, Detection and Tracking of Point Features //Technical Report CMU-CS-91-132 / School of Computer Science, Carnegie Mellon University. — April 1991. http://www.ri.cmu.edu/pubs/pub_2543.html

[11] J. Demmel. Applied Numerical Linear Algebra// SIAM, 1997.

[12] H.C. Longuet-Higgins. A Computer Algorithm for Reconstructing a Scene From Two Projections," Nature, vol. 293, pp. 133-135, Sept 1981.

[13] V.A. Kalinichenko, N.V. Sveshnikova, D.V. Yurin The Epipolar Geometry and its Accuracy from the Results of the Factorization Recovery. // In Conference Proceedings. 16-th International Conference on Computer Graphics and Application GraphiCon'2006 July 1-5, 2006 Novosibirsk Akademgorodok, Russia.G. O. Young, "Synthetic structure of industrial plastics (Book style with paper title and editor)," in Plastics, 2nd ed. vol. 3, J. Peters, Ed. New York: McGraw-Hill, 1964, pp. 15-64. 